\title{
Assessment of C-reactive protein and procalcitonin levels to predict infection and mortality in burn children
}

\author{
María Teresa Rosanova, M.D. ${ }^{a}$, Nidia Tramonti, M.D. ${ }^{b}$, Moira Taicz, M.D. ${ }^{a}$, \\ Soledad Martiren, M.D. ${ }^{a}$, Hugo Basílico, M.D. ${ }^{b}$, Cecilia Signorelli, M.D. ${ }^{b}$, \\ Ana Buchovsky, M.D. ', and Roberto Lede, M.D. ${ }^{d}$
}

\begin{abstract}
C-reactive protein (CRP) and procalcitonin (PCT) levels may distinguish between a systemic inflammatory response and an infection in burn children.

Objectives. To establish the operative capability of CRP and PCT to diagnose infections and mortality. Methods. Burn patients admitted to the hospital with clinical suspicion of an infection were included. CRP and PCT were measured and their operative diagnostic capabilities were calculated.

Results. Forty-eight patients (p) were included. Their median age was 49 months old (r: 17-86). The median burned surface area was $40 \%$ (r: 30$48 \%) ; 28 \mathrm{p} \mathrm{(58 \% )} \mathrm{had} \mathrm{type} \mathrm{AB}$ and type B burn wounds. Infection was confirmed in $32 \mathrm{p}(66.7 \%)$; the most common infection was burn-related sepsis $(24 \mathrm{p}, 75 \%)$, followed by burn wound infection $(6 \mathrm{p}, 19 \%)$. Eight patients (17\%) died. It was not possible to establish CRP sensitivity and specificity because it was high in all patients, regardless of mortality, survival or the presence of infection. In relation to infections, PCT had a $90.6 \%$ sensitivity $(95 \%$ confidence interval $[\mathrm{CI}]$ : 75.8-96.8\%), a $18.8 \%$ specificity (95\% CI: 6.6- $43 \%$ ), a $69 \%$ positive predictive value (PPV) and a $50 \%$ negative predictive value (NPV). In relation to 30-day mortality, sensitivity was 100\% (95\% CI: $67.6-100 \%$ ), specificity $15 \%$ (95\% CI: 7.1-29.1\%), PPV 19\% (95\% CI: $10-33.3 \%)$ and NPV 100\% (95\% CI: 61-100\%).

Conclusions. In pediatric burn patients, neither CRP nor PCT showed an adequate operative capability to detect an infection or a fatal outcome. Key words: burn patients, procalcitonin, C-reactive protein, children.
\end{abstract}

http:/ /dx.doi.org/10.5546/aap.eng.2015.36

\section{INTRODUCTION}

Infections are the main cause of death in burn patients. The overall incidence of infections is approximately $60 \%$, being sepsis and burn wound infections the most common ones. ${ }^{1}$

In spite of advances in relation to the management of burn patients, infections continue to be the main cause of morbidity and mortality in these patients due to the disruption of the skin barrier and the compromise of the immune system, which are common complications in hospitalized patients with thermal injuries..$^{2-4}$

Considering that the timely diagnosis and treatment of the infection are associated with a better clinical course, it is very important to have early markers. In these patients, the duration of treatment is not clearly defined; therefore, having a followup parameter of the inflammatory response might be potentially useful for treatment decisions. ${ }^{5-7}$

Quantitative C-reactive protein (CRP) is an inflammatory response marker, and when its plasma level is high, it is associated with the presence and progression of certain infections, but there are few studies that have been conducted in burn children.,

Other acute-phase reactant proposed to diagnose sepsis is procalcitonin (PCT). PCT levels increase 6-12 hours of the initial bacterial infection and decrease once the infection is controlled. This marker has been used to specifically distinguish between a bacterial infection and other causes of inflammatory response.

Based on the above mentioned information, the practical use of these markers may diminish empiric broad-spectrum antibiotic use, shorten antibiotic therapy duration, and reduce the development of antimicrobial resistance and health care costs. However, bibliographic evidence on the use of these markers to identify and/or diagnose infections specifically in pediatric burn patients is scarce. Therefore, we considered 
that communicating our experience on this subject would be of interest.

\section{OBJECTIVES}

To assess the operative capability of CRP and PCT to detect an infection in the first seven days following a burn injury and to predict the occurrence of death within 30 days of admission in acutely burn children from the community hospitalized with clinical suspicion of infection.

\section{MATERIAL AND METHODS}

1) Design: prospective and descriptive study.

The study was approved by the hospital's Teaching Committee. In all cases, the informed consent was obtained from the child's parents or legal tutors.

\section{2) Population}

2.1. Inclusion criteria: Patients $(p)$ older than 1 month old and younger than 18 years old from the community admitted to the Burn Unit between December 2011 and December 2013, with no history of hospitalization or prior antibiotic therapy, who had suffered an acute burn injury and with clinical suspicion of infection in the first week after having suffered burn injuries.

2.2. Exclusion criteria: Patients admitted to the Burn Unit for other reasons (e.g., postoperative patients, severe drug-induced skin disorders) or burn patients with more than one week of progression and/or receiving antibiotics at the time of admission to the Burn Unit and/or with a clinically or microbiologically documented infection were excluded.

3) Study procedures:

3.1. Patient inclusion: All patients who met eligibility criteria, treated in the study period were included. Clinical suspicion of infection was estimated by a doctor from the Plastic Surgery and Burn Unit. It was considered present if clinical and/or laboratory changes indicative of organ failure and/or local changes in the burn wound were observed. If this was the case, the physician would request the corresponding culture tests, blood collection and empiric antibiotic administration until bacteriologic results were available. In all cases, CRP and PCT tests were conducted at the time of inclusion into the study when infection was suspected.

3.2 Data collection: A patient follow-up card was developed to collect the following variables: age, burn injury type, burned surface area, Garces' index, infection and microorganism type, length of stay, and fatal outcome.

3.3 CRP measurement: The CRP value was measured using the particle-enhanced immunoturbidimetric method (Roche/ Hitachi-Cobas system). Values $\leq 5 \mathrm{mg} / \mathrm{L}$ were considered normal.

3.4 PCT measurement: PCT was measured using the Vidas Biomerieux immunoassay system. Values $\leq 0.5 \mathrm{ng} / \mathrm{mL}$ were considered normal.

3.5. Variable definition:

1) Age: in months old.

2) Burn wound type: superficial or $A$, intermediate or $\mathrm{AB}$, and deep or $\mathrm{B}$.

3) Burned surface area: percentage of body surface area.

4) Garces' index: this a severity and mortality prediction index calculated as follows: 40 minus patient's age, plus burn wound percentage, multiplied time 1 (if type $\mathrm{A}$ burn injury), times 2 (if type $A B$ burn injury), and times 3 (if type $B$ burn injury). From 0 to 60 points: grade 1 (mild risk). From 61 to 90 points: grade 2 (moderate risk). From 91 to 120 points: grade 3 (severe risk); over 121 points: grade 4 (critical risk).

5) Infection type: infections were defined as per the American Burn Association (ABA) criteria. ${ }^{7}$ Microbiological isolation had to be present in all cases.

6) Length of hospital stay.

\section{Outcome measures}

a) Primary: presence of bacteriologically documented sepsis and/or infections in the first seven days after the burn injury. It was established within the first week to reduce interference with hospital-acquired infections.

b) Secondary: death within 30 days of hospitalization. Death was considered related to infection if it occurred in the presence of positive culture tests and/or clinical infection.

Statistical analysis: A descriptive analysis was performed. Quantitative variables were expressed as median (mdn) and interquartile range (r). Categorical variables were expressed as percentages. Contingency tables were prepared, sensitivity, specificity and likelihood ratios (LR) of 
CRP and PCT values were calculated for the first sample to predict sepsis or other bacteriologically documented infection and 30-day mortality. Statistical analyses were conducted using the SPSS software for Windows, version 11.5.

\section{RESULTS}

Forty-eight patients were included. Table 1 shows population characteristics at the time of study inclusion. It can be observed that this was a cohort of pediatric burn patients with severe involvement, based on the burned surface area, burn wound depth and Garces' index.

Infection was confirmed in the cultures of $32 \mathrm{p}(66.7 \%)$; burn-related sepsis was the most common infection ( $24 \mathrm{p}, 75 \%)$, followed by burn wound infection $(6 \mathrm{p}, 19 \%)$. One patient had a catheter-associated urinary tract infection $(3 \%)$ and one (3\%) was diagnosed with pneumonia. The median length of stay in the hospital was 29.5 days (r: 15-52).

The prevalence of documented infection was $66.7 \%$, while the mortality incidence was $17 \%$, with $8 \mathrm{p}$ who died as a result of infection.

1. CRP: The median level at the time of admission was $96.7 \mathrm{mg} / \mathrm{L}$ ( $\mathrm{r}: 37,10$ $132,2)$. Sensitivity reached $100 \%$, but it was not possible to establish specificity and likelihood ratios because this marker was high in all patients, regardless of the presence of infection or subsequent death (Table 2, $a$ and $b$ ).

2. PCT: The median PCT level at the time of admission was $1.97 \mathrm{ng} / \mathrm{mL}$ (r: 1.15-7). Sensitivity for infection was $90.6 \%$ (95\% confidence interval [CI]: 75.8-96.8), while specificity was $18.8 \%$ (95\% CI: 6.6-43.0). LR+ was 1.12 (95\% CI: 0.86-1.45) and LR- was 0.50 (95\% CI: 0.14-1.74). Patients who died had an elevated PCT at the time of admission, so the

TABle 1. Patients characteristics ( $n: 48 p$ )

\begin{tabular}{lc}
\hline Characteristics & Description \\
\hline Age, months old & M $29(\mathrm{r}: 17-75)$ \\
Male & $60 \%(\mathrm{n}: 29)$ \\
Burned surface area & md $40 \%(\mathrm{r}: 30-48)$ \\
AB or B depth & $58 \%(\mathrm{n}: 28)$ \\
Garces' index & md 4 (r: $1-4)$ \\
\hline
\end{tabular}

M: months old.

r: range.

md: median. sensitivity of the method for this endpoint increases to $100 \%$ (95\% CI: $67.6-100)$, but its specificity only reaches $15.0 \%$ (95\% CI: 7.1-29.1). In relation to infection, the LR+ reached 1.12 and the LR- was 0.5 . In relation to mortality, the LR+ was 1.18 , while the LRwas 0 (Table $3, a$ and $b$ ).

\section{DISCUSSION}

Severe burn wounds induce a state of immunosuppression that predisposes patients to infectious complications. ${ }^{8}$ Burn patients is the most comprehensive and complex model of the inflammatory process, where all inflammation mediators are present with disruption of homeostasis and multiple organ

TABLE 2a. Contingency table of C-reactive protein values and presence of infection

\begin{tabular}{lccc}
\hline CRP & & & \\
Infection & Infected & Not infected & Total \\
\hline High & 32 & 16 & 48 \\
Normal & 0 & 0 & 0 \\
Total & 32 & 16 & 48 \\
\hline
\end{tabular}

\begin{tabular}{lcc} 
& \multicolumn{1}{c}{$\mathbf{9 5 \%}$ confidence interval } \\
\hline Sensitivity & LR- $100.0 \%$ & $89.3 \%-100.0 \%$ \\
Specificity & 0 & \\
LR+ & Not quantifiable & \\
LR- & Not quantifiable & \\
\hline
\end{tabular}

CRP: C-reactive protein.

LR: likelihood ratio.

TABLE $2 \mathrm{~b}$. Contingency table of C-reactive protein values and mortality

\begin{tabular}{lccc}
\hline $\begin{array}{l}\text { CRP } \\
\text { Mortality }\end{array}$ & Dead & Alive & Total \\
\hline High & 8 & 40 & 48 \\
Normal & 0 & 0 & 0 \\
Total & 8 & 40 & 48 \\
\hline
\end{tabular}

CRP: C-reactive protein.

\begin{tabular}{lcc} 
& \multicolumn{2}{c}{$95 \%$ confidence interval } \\
\hline Sensitivity & $100.0 \%$ & $67.6 \%-100.0 \%$ \\
Specificity & 0 & \\
LR+ & Not quantifiable \\
LR- & Not quantifiable & \\
\hline
\end{tabular}

LR+: positive likelihood ratio.

LR-: negative likelihood ratio. 
dysfunction. ${ }^{9}$ Burn patients are chronically exposed to inflammatory mediators and develop a systemic inflammatory response syndrome with signs and symptoms similar to sepsis, therefore rendering the differential diagnosis difficult. Burn patients with no infection may even develop fever or hypothermia, tachypnea, tachycardia, leukopenia or leukocytosis as part of the systemic inflammatory response syndrome. This leads to an inadequate antibiotic use, which in turn translates into increased antibiotic resistance and hospital costs. ${ }^{10}$

When a burn injury occurs, a systemic inflammatory response takes places due to the release of cytokines, prostaglandins and oxygen radicals from the burn site. The burn wound increases capillary permeability and

TABLE 3a. Contingency table and operative capability of procalcitonin levels for infection

\begin{tabular}{lccc}
\hline $\begin{array}{l}\text { PCT } \\
\text { Infection }\end{array}$ & Infected & Not infected & Total \\
\hline High & 29 & 13 & 42 \\
Normal & 3 & 3 & 6 \\
Total & 32 & 16 & 48 \\
\hline
\end{tabular}

PCT: procalcitonin.

\begin{tabular}{lcc} 
& & $\mathbf{9 5 \%}$ confidence interval \\
\hline Sensitivity & $90.6 \%$ & $75.8 \%-96.8 \%$ \\
Specificity & $18.8 \%$ & $6.6 \%-43.0 \%$ \\
LR+ & 1.12 & $0.86-1.45$ \\
LR- & 0.50 & $0.14-1.74$ \\
\hline
\end{tabular}

LR+: positive likelihood ratio.

LR-: negative likelihood ratio.

TABLE 3b. Contingency table and operative capability of procalcitonin levels for mortality prediction

\begin{tabular}{lccc}
\hline PCT & & & \\
Mortality & Dead & Alive & Total \\
\hline High & 8 & 34 & 42 \\
Normal & 0 & 6 & 6 \\
Total & 8 & 40 & 48 \\
\hline
\end{tabular}

\begin{tabular}{lcc} 
& & $\mathbf{9 5 \%}$ confidence interval \\
\hline Sensitivity & $100.0 \%$ & $67.6 \%-100.0 \%$ \\
Specificity & $15.0 \%$ & $7.1 \%-2901 \%$ \\
LR+ & $1.10 \%$ & $1.03-1.34$ \\
LR- & 0.00 & \\
\hline
\end{tabular}

PCT: procalcitonin.

$\mathrm{LR}+$ : positive likelihood ratio.

LR-: negative likelihood ratio. interstitial fluid extravasation occurs. When the burned surface area is larger than $40 \%$, myocardial depression and secondary shock may also take place. In patients with major burn wounds, there is usually hypermetabolism, which increases protein catabolism. All these mediators are responsible for the systemic inflammatory response syndrome. ${ }^{11}$ Septic patients experience multiple physiological changes, e.g., fever and increased fluid requirements, as well as liver, kidney and lung failure. To this date, no laboratory test has proven to be reliable enough to distinguish between an inflammatory response typical of a burn wound and sepsis. ${ }^{12}$

In a study conducted by Lobo, et al., ${ }^{13}$ it was established that severe patients who have high levels of CRP at the time of admission to the intensive care unit required a prolonged stay and had a higher mortality rate. Those patients whose CRP continued elevated at 48 hours had a significantly higher mortality than those whose levels reduced. Burn patients were considered a subgroup in this study population. Other series of burn patients showed that high CRP levels were correlated to a higher mortality risk, while persistently high levels were related to a worse clinical course. ${ }^{14}$

Housinger, et al. ${ }^{15}$ studied post-burn changes in physiological parameters, such as white blood cell count, platelet count, temperature and fluid requirements. They concluded that a reduction in platelet count to less than $100000 / \mathrm{mm}^{3}$ predicts pulmonary, blood and cardiovascular changes associated with sepsis. Other authors suggest that changes in CRP levels measured every day are useful to predict sepsis even earlier than a platelet count. ${ }^{16}$ In our series, all patients had increased CRP, regardless of the confirmation of sepsis and/or other infections, which remained high in all protocol measurements. Given that this prevalence reached $66.6 \%$, it is obvious that the PPV $(62.5 \%)$ of CRP was of no clinical utility. As in our series, Jeschke, et al. ${ }^{17}$ concluded that $\mathrm{CRP}$ is not an adequate infection or sepsis marker and only reflects the inflammatory mechanisms triggered by thermal injuries.

Procalcitonin is the precursor peptide of calcitonin and is released at any body site in response to bacterial toxins and specific bacterial proinflammatory mediators, such as interleukin$1 \mathrm{~b}$, tumor necrosis factor and interleukin. ${ }^{18}$ Persistent high values are associated with the presence of a bacterial infection. ${ }^{19,20}$ Procalcitonin high negative predictive value may allow to 
exclude antibiotic use and decide on antibiotic therapy maintenance, discontinuation or modification in critical patients. ${ }^{21}$ However, in certain situations (newborn infants, patients with multisystem trauma, burn wounds, major surgery or severe cardiogenic shock), a high CRP may occur regardless of any infection attack. ${ }^{22}$

In the study by Barati, et al. ${ }^{5}$ it was observed that PCT levels were significantly higher in burn patients with systemic infections than in burn patients with no infection. Values higher than $0.5 \mathrm{ng} / \mathrm{mL}$ showed a $100 \%$ sensitivity and a $83.3 \%$ specificity to diagnose infections, so they concluded that PCT is highly predictive of infection. In our series, all patients except six (three with infection and three with no infection) had a PCT level $>0.5 \mathrm{ng} / \mathrm{mL}$; therefore, sensitivity and specificity in this study were not comparable to that of Barati, et al.

Lavrentieva, et al. ${ }^{23}$ indicated that the significance of PCT in the diagnosis of infectious complications in burn patients is controversial, mostly due to the inconsistent definitions of sepsis among the different studies. Although in their series high PCT values had certain prognostic value for infectious complications and for monitoring treatment, they concluded that more studies are required to assess whether mortality and costs would be reduced if this biomarker was used for the early detection of infections. Seoane, et al. ${ }^{20}$ suggested that, in adult burn patients, PCT may not be an accurate sepsis marker, and this is similar to what has been observed in our pediatric series. Mann, et al. ${ }^{21}$ made a systematic review of the literature and concluded that, although PCT may be useful to diagnose sepsis in burn patients, there is inconsistency in the findings of certain studies because varying definitions of sepsis were used and the cost of this method may restrict its availability. Most studies were conducted in adult populations. In the aforementioned systematic review, Neely, et al. ${ }^{18}$ concluded, based on 20 pediatric patients, that PCT was not as effective for the early diagnosis of sepsis as a platelet count and a CRP serial measurement. PCT sensitivity was $42 \%$, and its specificity, $67 \%$.

In spite of the advances made in the management of burn patients, mortality is still high. In our series, mortality reached $15 \%$ and, in all cases, was related to the presence of infection. Neither PCT nor CRP levels were helpful to identify patients with a higher risk of death. The almost null specificity of the test in relation to this endpoint renders it empirically inapplicable, as observed in Baratti, et al.'s study ${ }^{5}$ but inconsistent with the findings of Lobo, et al. in critically ill patients, where burn subjects were considered a subgroup. ${ }^{13}$

Given the high prevalence of infections in these patients, non-optimal predictive tests result of little clinical utility because they cannot substantially modify the early probability of occurrence. In these situations (high prevalence), more specific tests are required, as opposed to sensitive tests (it is more important to rule out the endpoint than to confirm its presence since, given its high frequency, it is likely to be present). Based on the high prevalence of this event and the inaccuracy of the test, it would be advisable to provide empirical therapy if infections and/or sepsis are suspected, because incorrectly ruling them out would put patients at a higher risk. For a major endpoint such as the presence of infection, it is not advisable to use the CRP test, which has a $23.5 \%$ rate of estimated false negative results (one in four patients with a negative CRP for infection will develop an infection). In addition, all patients who died had a high CRP, but none of those who survived had normal values. PCT did not show a useful operative capability either, since it did not successfully identify those who would not develop an infection (it predicted only one in six) or those who would die (it predicted only one in seven).

One of the limitations of the study is that it was conducted in patients hospitalized in a large referral center for major burn patients, so these results may not be extrapolated to less severe burn wounds. It is assumed that, in patients with major burn wounds, an excessive inflammatory response to thermal injuries is the cause of positive biomarkers, regardless of infection, 30day survival or mortality, which may mask its operative capability in patients with less severe burn wounds. It should be noted that, although this was a relatively small series to make highly powerful statistical conclusions, as per our knowledge, it is the largest series published so far on this issue.

\section{CONCLUSIONS}

The tests assessed in this study did not show an adequate operative capability in patients with severe burn wounds so as to promote their clinical application to detect which patients will develop an infection or a fatal outcome. 


\section{REFERENCES}

1. Pruitt BA Jr, McManus AT. The changing epidemiology of infections in Burn patients. World J Surg 1992;16(1):57-67.

2. Rosanova MT, Stamboulian D, Lede R. Infecciones en los niños quemados: análisis epidemiológico y de los factores de riesgo. Arch Argent Pediatr2013;111(4):303-8.

3. Mayhall CG. The epidemiology of burn wound infections: then and now. Clin Infect Dis 2003;37(4):543-50.

4. Rosanova MT, Stamboulian D, Lede R. Risk factors for mortality in burn children. Braz J Infect Dis 2014;18(2):144-9.

5. Barati M, Alinejad F, Bahar MA, Tabrisi MS, et al. Comparison of WBC, ESR, CRP and PCT serum levels in septic and non-septic burn cases. Burns 2008;34(6):770-4.

6. Povoa P. C-reactive protein: a valuable marker of sepsis. Intensive Care Med 2002;28(3):235-43.

7. Greenhalgh DG, Saffle JR, Holmes JH 4th, Gamelli RL, et al. American Burn Association consensus conference to define sepsis and infections in burns. J Burn Care Res 2007;28(6):776-90.

8. Geyik MF, Aldemir M, Hosoglu S, Tacyildiz HI. Epidemiology of burn unit infections in children. $A m \mathrm{~J}$ Infect Control 2003;31(6):342-6.

9. Heideman M, Bengstsson A. The immunologic response to thermal injury. World J Surg 1992;16(1):53-6.

10. Sheridan RL. Sepsis in pediatric burn patients. Pediatr CritCare Med 2005;6(3 Suppl):112-9.

11. Murray CK, Hoffmaster RM, Schmit DR, Hospenthal DR, et al. Evaluation of white blood cell count, neutrophil percentage, and elevated temperature as predictors of bloodstream infection in burn patients. Arch Surg 2007;142(7):639-42.

12. Von Heimburg D, Stieghorst W, Khorram-Sefat R, Pallua N. Procalcitonin: a sepsis parameter in severe burn injuries. Burns 1998;24(8):745-50.

13. LoboSM,LoboFR, Bota DP,Lopes-FerreiraF, etal.C-reactive protein levels correlate with mortality and organ failure in critically ill patients. Chest 2003;123(6):2043-9.

14. BelgianOutcomein Burn Injury Study Group. Development and validation of model for prediction of mortality in patients with acute burn injury. Br J Surg2009;96(1):111-7.

15. Housinger TA, Brinkerhoff $C$, Warden GD. The relationshipbetweenplatelet count, sepsis, and survival in pediatric burn patients. Arch Surg 1993;128(1):65-6; discussion 66-7.

16. Jaye DL, Waites KB. Clinical applications of C-reactive protein in pediatrics. Pediatr Infect Dis J 1997;16(8):735-46; quiz 746-7.

17. Jeschke MG, Finnerty CC, Kulp GA, Kraft R, et al. Can we use C-reactive protein levels to predict severe infection or sepsis in severely burned patients? Int J Burn Trauma 2013;3(3):137-43.

18. Neely AN, Fowler LA, Kagan RJ, Warden GD. Procalcitonin pediatric burn patients: an early indicator of sepsis? J Burn Care Rehabil 2004;25(1):76-80.

19. Castelli GP, Pognani C, Meisner M, Stuani A, et al. Procalcitonin and C-reactive protein during systemic inflammatory response syndrome, sepsis and organ dysfunction. Crit Care 2004;8(4):R234-42.

20. Seoane L, Pertega S, Galeiras R, Astola I, et al. Procalcitonin in the burn unit and the diagnosis of infection. Burns 2014;40(2):223-9.

21. Mann EA, Wood GL, Wade CE. Use of procalcitonin for the detection of sepsis in the critically ill burn patient: a systematic review of the literature. Burns 2011;37(4):549-58.

22. Kim HS, Yang HT, Hur J, Chun W, et al. Procalcitonin levels within 48 hours after burn injury as a prognostic factor. Ann Clin Lab Sci 2012;42(1):57-64.

23. Lavrentieva A, Papadopoulou S, Kioumis J, Kaimakamis E, et al. PCT as a diagnosic and prognostic tool in burn patients. Whether time course has a role in monitoring sepsis treatment. Burns 2012;38(3):356-63. 